\title{
ER:YAG LASER APPLICATION IN ORTHODONTIC PRACTICE. A CASE REPORT
}

\author{
Svetlana Yordanova ${ }^{1}$, Miroslava Yordanova $^{1}$, G. Tomov $^{2}$, Hristina Lalabonova $^{3}$ \\ ${ }^{1}$ Department of Orthodontics, \\ ${ }^{2}$ Department of Operative Dentistry and Endodontics, \\ ${ }^{3}$ Department of Maxillofacial Surgery, \\ Faculty of Dental Medicine, Medical University - Plovdiv, Bulgaria
}

\begin{abstract}
Introduction: Er:YAG lasers generate wave length of $2940 \mathrm{~nm}$ which coincides with the absorptions spectrum of water. For these reasons it is possible to work with Er:YAG lasers both on soft and hard tissues with minimal thermal effects. Lasers due to their wide-spectrum of biological action and high therapeutic effectiveness are used long ago in certain specialties like oral surgery and cariesology. In orthodontic lasers are poorly known and are not routine tool despite their beneficial application abilities.

Aim: The presented case report discusses alternative surgical approach for laser-assisted uncovering of ectopically impacted canines for orthodontic reasons.

Conclusions: Er:YAG laser is a revolutionary technology providing alternatives for orthodontists in solving different problems in their everyday practice. It is effective and comfortable tool to reduce treatment time and to promote excellent clinical results. To patients is offered method of choice with comfort guarantee, less pain sensations and accelerated healing process
\end{abstract} LiteTouch

Key words: Orthodontic treatment, Er:YAG laser

The orthodontic industry constantly present new tools, methods and technologies for better treatment. Their aim is to reduce the undesirable side effects, to enhance and accelerate achieving of desired treatment results. Today more and more patients insist on shorter treatment duration and for these reasons the orthodontist is challenged to accelerate the treatment procedure and to shorten the active treatment time.

Lasers due to their wide-spectrum of biological action and high therapeutic effectiveness are used for decades in certain specialties like oral surgery and periodontology $(1,2)$. In orthodontic lasers are poorly known and they are not used in routine practice despite their benefit. The Er:YAG laser is a hard corps laser with active media of Itrium-Aluminium Granate steed with Erbium (Lantanoid metals group) (3). It is introduced recently in dental practice. Er:YAG lasers generate wave length of $2940 \mathrm{~nm}$ which coincides with the absorptions spectrum of water and for these reasons it is possible to work with Er:YAG lasers both on soft and hard tissues with minimal thermal effects and without damaging dental pulp $(4,5,6,7)$.

In 2009 on the market was presented first non-fiber, all-tissue Er:YAG dental laser incorporated in the handpiece (LiteTouch, Syneron, Israel) (8). LiteTouch comes with a variety of long and short tips, and with diameters ranging from 200 to 1300 microns. Offering great convenience to the dentist, these different tips can be employed in different parts of the mouth, and in different hard and soft tissue procedures (gingivectomy, operculectomy, frenectomy, osteotomy, laser etching etc). The hemostatic effect is lower compared with diodes lasers, but this is regarding to minimal thermal trauma in tissues, faster regeneration and minimal postoperative discomfort. The use of anesthesia is reduced and depends on intervention depth.

Er:YAG lasers with their benefits could be applied in every single step of the complex orthodontic treatment. They could be effective treatment modality before, during and post- treatment when the orthodontic devices are removed.

AIM.

The presented case report discusses alternative approach of laser-assisted tooth exposure with Er:YAG laser Lite Touch for orthodontic reason.

The patient N.Hr aged 11 years had been treated orthodonticaly by fixed appliance MBT 0.22 on the upper jaw (Fig. 1). The brackets bonding on teeth 13 and 23 was impossible due to their unfinished eruption and presence of gingival tissue covering the vestibular side of their crowns. For these reasons their including in the first-step treatment plan failed. The ectopic position of 13 was also obstacle for fixing bracket on the neighbor 12 tooth. As a result the leveling and alignment of the rest teeth was impede. To wait for natural eruption of teeth 13 and 23 means several months of no effective and prolonging treatment term and after 
parent's consent laser-assisted uncovering was done by Er:YAG laser LiteTouch (Fig. 2). The laser surgery provided fast postoperative recovery and no pain or administration of drugs were reported. This procedure allows in shorter period (10 days after) to fix brackets on teeth 13 and 23 and to initiate their movement (Fig. 3). The leveling and alignment phase are in progress. The faster alignment of 13 and 23 will accelerate the finishing phase.
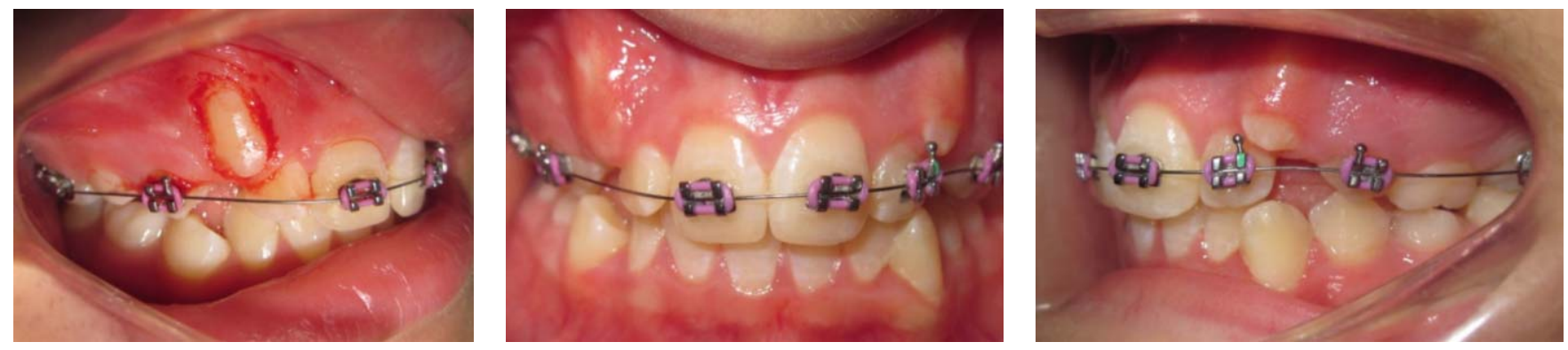

Fig. 1. Intraoral view of patient N. Hr. (11 years old female) at the beginning of orthodontic treatment
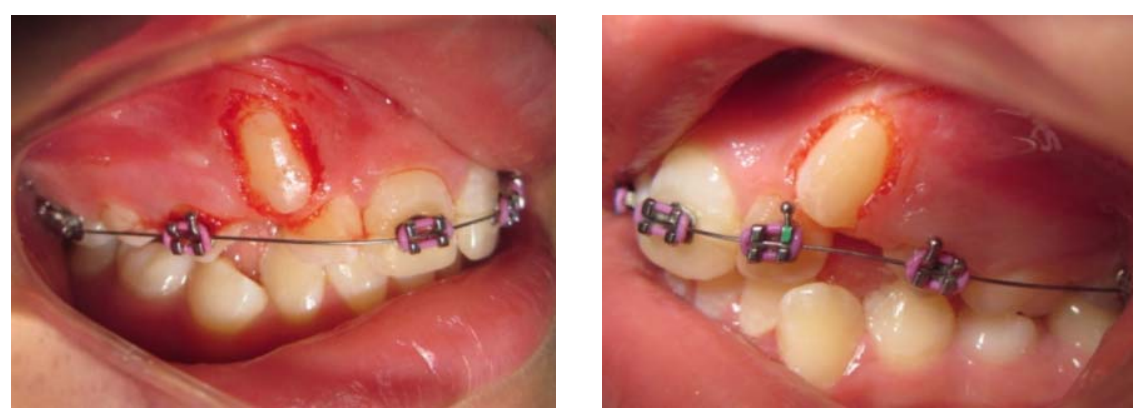

Fig. 2. Laser-assisted exposure of 13 and 23 teeth
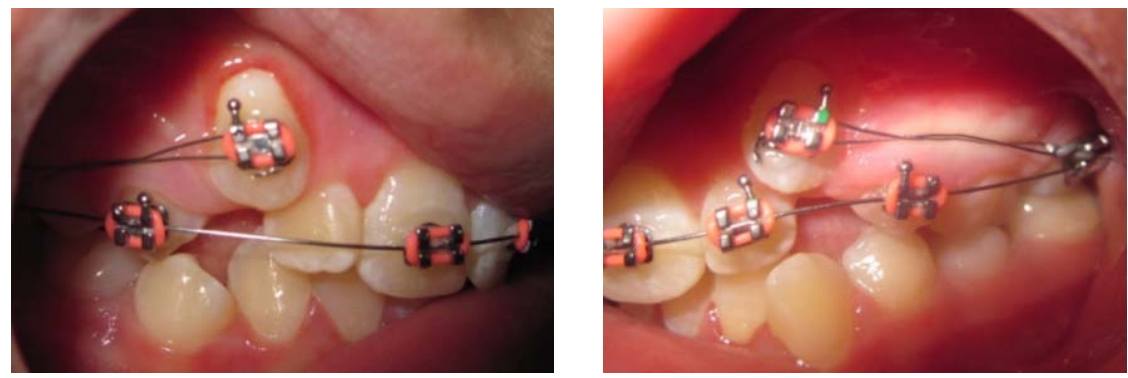

Fig. 3. Brackets fixation 10 days after laser-assisted exposure

\section{DISCUSSION.}

Usually the canines are the teeth last involved in orthodontic alignment due to their slower and subsequent passive eruption or retention in the bone. The waiting for natural eruption prolonged the leveling phase and it is accompanied with long period of thin and elastic wires presence, multiple brackets rebonding or bending wires for gradual canines alignment. This situation is connected with additional patient's visits, time- consuming procedures and prolonged treatment.

The surgical exposure is usually performed by conventional surgical methods, which are related to pain, inflammation and fear of scalpel by majority of patients. To reduce all these undesirable effects, in this clinical case alternative approach of laser-assisted tooth exposure with Er:YAG laser Lite Touch was preferred for this additional surgical procedure. 


\section{CONCLUSIONS.}

Er:YAG laser is a revolutionary technology providing alternatives for orthodontists in solving different problems in their everyday practice. It is effective and comfortable modality to reduce treatment time and to promote excellent clinical results. To patients is offered method of choice with comfort guarantee, less pain sensations and accelerated healing process.

\section{REFERENCES:}

1. Sulewski JG. Historical survey of laser dentistry. Dent Clin North Am 2000 Oct:44(4):717-52. [PubMed]

2. Wigdor HA, Walsh JT Jr, Featherstone JD, Vlsuri SR, Fried D, Waldvogel JL. Lasers in dentistry. Lasers Surg Med. 1995;16(2):103-133. [PubMed]

3 . Lalabonova Hr, Firkova E. Lasers in dentistry. Physical. Medicine. Rehabilitation. Health. 2005; 4:4-9.

4. Hibst R, Keller U. Experimental studies of the application of the Er: YAG laser on dental hard substances. I. Measurement of the ablation rate. Lasers Surg Med. 1989; 9(4):338-344. [PubMed] 5. Ishikawa I, Sasaki KM, Aoki A, Watanabe H. Effects of Er: YAG laser on periodontal therapy. J Int Acad Periodontol 2003 Jan;5(1): 23-28. [PubMed]

6. Keller U, Hibst R. Experimental studies of the application of the Er: YAG laser on dental hard substances. II. Light microscopic and SEM investigations. Laser Surg Med. 1989: 9(4):345-351. [PubMed] 7. Prachis $\mathrm{Ph}$, Prashanth K, Renu P, Vishwanath. Lasers - a contemporary tool in orthodontics. J Int Oral Health 2011; 3(2): $15-21$.

8. Tomov G.. Laser surgery in everyday practice with Er: YAG laser Lite Touch. Dental Tribune (Bulgarian edition), 2011; Vol. 9(5):6-7.

\section{Address for correspondence:}

Dr. Svetlana Yordanova

Department of Orthodontics, Faculty of Dentistry, Medical University, Plovdiv

2, Hristo Botev Str., 4000 Plovdiv, Bulgaria

Mobile: 00359878147640,

E-mail: svetlaj@gmail.com 